\title{
ON THE GALOIS COHOMOLOGY OF THE RING OF INTEGERS IN AN ALGEBRAIC NUMBER FIELD
}

\author{
M. P. LEE AND M. L. MADAN
}

Notation. $Z=$ the ring of rational integers,

$Q=$ the field of rational numbers,

$K=$ a field of algebraic numbers of finite degree over $Q$,

$F=$ a finite normal extension of $K$,

$O_{K}, O_{F}=$ the ring of all integers of $K, F$ respectively,

$G=$ Galois group of $F$ over $K$.

Introduction. $G$ operates in a natural way on the additive groups of $F$ and $O_{F}$. It is well known that $H^{r}\left(G, F^{+}\right)$, the $r$-dimensional cohomology group of $G$ with $F^{+}$as coefficients-module is trivial for all integer values of $r$. In [6]- [9] Yokoi has obtained the following results concerning $H^{r}\left(G, O_{F}^{+}\right)$:

|THEOREM I. If the 0-dimensional cohomology group $H^{0}\left(G, O_{F}\right)$ is trivial, (we write $O_{F}$ for $\left.O_{F}^{+}\right)$, then $H^{r}\left(V, O_{F}\right)$ is trivial in all dimensions for all subgroups $V$ of $G$.

Theorem II. If $G$ is cyclic of prime order, the groups $H^{r}\left(G, O_{F}\right)$ are isomorphic in all dimensions.

THEOREM III. If $G$ is arbitrary cyclic, all the groups $\operatorname{Hr}^{r}\left(G, O_{F}\right)$ have the same order.

On the basis of these results he conjectured in [9] that the groups $H^{r}\left(G, O_{F}\right)$ have the same order also in the case when $G$ is not cyclic. In the present note, we shall show that the conjecture is false. We shall also demonstrate how the problem of determining $H^{r}\left(G, O_{F}\right)$ can be localized. In the end, we shall make some remarks concerning proofs of Theorems I, II and III and give a generalization of Theorem $\mathrm{I}$ in the case where $G$ is nilpotent.

Counterexample. Let $K=Q, F$ be the splitting field of $f(x)=x^{3}-2$ over $K, \theta$ be the real root of $f(x)$ and $E=Q(\theta) . F=E(\eta)$, where $\eta$ is a primitive 3rd root of unity. $G$ is generated by two elements $\sigma$ and $\tau$ satisfying the generating relations $\sigma^{3}=\tau^{2}=1, \sigma^{2} \tau=\tau \sigma$. The action of $G$ on $F$ is given by: $\sigma(\theta)=\theta \eta, \sigma(\eta)=\eta, \tau(\theta)=\theta, \tau(\eta)=-1-\eta$.

We shall first find an integral basis of $F$ over $K . O_{E}$, the ring of all integers in $E$, is a principal ideal domain having a $Z$-basis consisting

Received by the editors September 1, 1967. 
of $1, \theta, \theta^{2}$. Consider the $O_{E}$-module $M=O_{E}+O_{E} \beta$, where $\beta$ $=(2+\eta)(1+\theta)^{-1}$. $\beta$ satisfies the equation $x^{2}-\left(\theta^{2}-\theta+1\right) x+\left(\theta^{2}-1\right)=0$ and so $\beta$ is in $O_{F}$. It can be easily verified that the relative discriminant of $M$ is $(1+\theta) O_{E}$. $\operatorname{Norm}_{E / \mathbb{Q}}(1+\theta)=3$ implies that $1+\theta$ is a prime element of $O_{E}$. Thus $\{1, \beta\}$ is an integral basis of $F$ over $E[2$, p. 129] and hence $\left\{1, \theta, \theta^{2}, \beta, \beta \theta, \beta \theta^{2}\right\}$ is an integral basis of $F$ over $K$.

It is now a simple matter to see that the set $\left\{b_{i}: i=1, \cdots, 6\right\}$, where $b_{1}=\theta+\theta^{2}, b_{2}=\left(\theta^{2}-2 \theta+\theta \beta-2 \beta\right), b_{3}=\theta, b_{4}=\theta \beta+\theta^{2} \beta-2 \theta, b_{5}=1$, $b_{6}=\beta-\theta^{2}$, is a $Z$-basis for $O_{F}$. The action of $G$ on $O_{F}$ is given by the table:

\begin{tabular}{|c|c|c|c|c|c|}
\hline$b_{1}$ & $b_{2}$ & $b_{3}$ & $b_{4}$ & $b_{5}$ & $b_{6}$ \\
\hline$b_{2}$ & $-b_{1}-b_{2}$ & $b_{4}$ & $-b_{3}-b_{4}$ & $b_{5}$ & $b_{6}+b_{1}$ \\
\hline$b_{1}$ & $-b_{1}-b_{2}$ & $b_{3}$ & $-b_{3}-b_{4}$ & $b_{5}$ & $b_{5}-b_{6}-b_{1}$ \\
\hline
\end{tabular}

Let $\alpha=\sum_{i=1}^{6} \alpha_{i} b_{i} \in O_{F}$. The trace of $\alpha, N(\alpha)=3\left(2 \alpha_{5}+\alpha_{6}\right) b_{5}$. Therefore $H^{0}\left(G, O_{F}\right) \cong\left(O_{F}^{G}\right) / N\left(O_{F}\right) \cong Z_{3}$. Let us now examine $H^{1}\left(G, O_{F}\right)$. If $h: G \rightarrow O_{F}$ is any 1 -cocycle, the group relations of $G$ yield the conditions: $(\tau+1) h(\tau)=0,\left(\sigma^{2}+\sigma+1\right) h(\sigma)=0$ and $(\sigma \tau+1)(h(\tau)-h(\sigma))=0$. These conditions imply $h(\tau)=x_{1} b_{1}+\left(2 x_{5}+2 x_{1}\right) b_{2}+x_{3} b_{3}+2 x_{3} b_{4}+x_{5} b_{5}$ $+\left(-2 x_{5}\right) b_{6}$ and $h(\sigma)=y_{1} b_{1}+\left(3 x_{1}+4 x_{5}-y_{1}\right) b_{2}+y_{3} b_{3}+\left(3 x_{3}-y_{3}\right) b_{4}$, where $x_{1}, x_{3}, x_{5}, y_{1}, y_{3} \in Z$. Choose $\alpha \in O_{F}$ as follows:

$$
\alpha=\sum_{i=1}^{6} \alpha_{i} b_{i}, \quad \alpha_{1}=2 x_{5}+x_{1}-y_{1}, \quad \alpha_{2}=-x_{5}-x_{1}, \quad \alpha_{3}=x_{3}-y_{3},
$$

$\alpha_{4}=-x_{3}, \alpha_{6}=x_{5}$ and $\alpha_{5}$ may be chosen arbitrarily. A simple calculation shows that $h(\tau)=(\tau-1) \alpha$ and $h(\sigma)=(\sigma-1) \alpha$. Thus every 1 -cocycle is a coboundary and $H^{1}\left(G, O_{F}\right)=0$. Yokoi's conjecture is thereby disproved.

Localization. For a prime divisor $\mathfrak{p}$ of $K$, let $\mathfrak{A}$ be a fixed prime divisor of $F$ lying above p. Let $O \mathfrak{Q}$ be the ring of integers of $F \mathfrak{Q}$, the completion of $F$ at $\mathfrak{A}$, and $G \mathfrak{Q}$ be the local group. We have the following:

\section{ThEOREM 1. $H^{r}\left(G, O_{F}\right) \cong \prod_{\mathfrak{p}} H^{r}\left(G_{\mathfrak{Q}}, O_{\mathfrak{Q}}\right)$ for all integers $r$.}

Proof. Let $\widetilde{O}_{F}=\prod_{\mathfrak{B}} O_{\mathfrak{B}}, \widetilde{O}_{K}=\prod_{\mathfrak{p}} O_{\mathfrak{p}}$, the first product taken over all prime divisors $\mathfrak{B}$ of $F . O_{F}$ is diagonally embedded in $\widetilde{O}_{F}$. Also $\widetilde{O}_{K}$ is canonically embedded in $\widetilde{O}_{F}$. Let $O^{(\mathfrak{p})}=\prod_{\mathfrak{P} / \mathfrak{p}} O_{\mathfrak{p}} . O^{(\mathfrak{p})}$ is $G$-module. 
By Shapiro's well-known lemma, $H^{r}\left(G, O^{(\mathfrak{p})}\right) \cong H^{r}\left(G_{\mathfrak{Q}}, O_{\mathfrak{Q}}\right)$. Now $\tilde{O}_{F} \cong \prod_{\mathfrak{p}} O^{(\mathfrak{p})}$. Therefore $H^{r}\left(G, \widetilde{O}_{F}\right) \cong \prod_{\mathfrak{p}} H^{r}\left(G, O^{(\mathfrak{p})}\right) \cong \prod_{\mathbb{Q}} H^{r}\left(G_{\mathbb{Q}}, O_{\mathbb{Q}}\right)$. Thus the isomorphism we wish to establish is equivalent to $H^{r}\left(G, \widetilde{O}_{F}\right)$ $\cong H^{r}\left(G, O_{F}\right)$, for all $r$. Let $[F: K]=n$ and $w_{1}, \cdots, w_{n}$ be a normal basis of $F$ over $K$ consisting of integers. Let $\mathfrak{M}=O_{K} w_{1}+\cdots+O_{K} w_{n}$. The index $\left[O_{F}: \mathfrak{M}\right]=l$ is finite. Therefore $\mathfrak{M}$ contains the ideal $\mathfrak{A}=(l)$ of $O_{F} . \mathfrak{A} \tilde{O}_{F} \subset \tilde{\mathfrak{M}}=\widetilde{O}_{K} w_{1}+\cdots+\tilde{O}_{K} w_{n}$. Therefore $\mathfrak{A} \widetilde{O}_{F}+O_{F}$ $\subset \tilde{\mathfrak{M}}+O_{F}$. But $\mathfrak{A} \tilde{O}_{F}+O_{F}=\tilde{O}_{F}\left[5\right.$, p. 195]. Therefore $\tilde{\mathfrak{M}}+O_{F}=\tilde{O}_{F}$. Thus

$$
\frac{\tilde{O}_{F}}{\tilde{\mathfrak{M}}} \cong \frac{\tilde{\mathfrak{M}}+O_{F}}{\tilde{\mathfrak{M}}} \cong \frac{O_{F}}{O_{F} \cap \tilde{\mathfrak{M}}} \cong \frac{O_{F}}{\mathfrak{M}}
$$

These are module-isomorphisms. $\mathfrak{M}, \tilde{\mathfrak{Q}}$ being $G$-regular, their cohomology is trivial. Therefore

$$
H^{r}\left(G, \widetilde{O}_{F}\right) \cong H^{r}\left(G, \widetilde{O}_{F} / \tilde{\mathfrak{M}}\right) \cong H^{r}\left(G, O_{F} / \mathfrak{M}\right) \cong H^{r}\left(G, O_{F}\right) .
$$

This completes the proof of Theorem 1.

Remarks. 1. A shorter and simpler proof of Theorem 1, which is also valid in a more general situation, can be constructed in the following way: Triviality of $H^{0}\left(G, O_{F}\right)$ is equivalent to the existence of an element $\alpha$ in $O_{F}$ of trace 1. The endomorphism $\phi_{\alpha}$ of $O_{F}$ defined by $\phi_{\alpha}(\beta)=\alpha \beta$ has the identity mapping as its trace. $\operatorname{Hr}\left(G, O_{F}\right)=0$ follows from a very elementary result $[1$, p. 18 , Satz 11$]$ in the cohomology theory of finite groups. To show $H^{r}\left(V, O_{F}\right)=0$ for any subgroup $V$ of $G$, we note trace $(\alpha)=1$ implies $\gamma=\sum \sigma_{j}(\alpha)$ has trace 1 w.r.t. $V$, where $\sigma_{j}$ is a representative system of right-cosets.

2. For proving Theorem II, it is enough to show that $H^{0}\left(G, O_{F}\right)$, $H^{1}\left(G, O_{F}\right)$ have the same order because any element of the cohomology group other than identity is of order $p$. Theorem II follows from Theorem III or from a theorem of Tate [3, p. 57, Theorem 10.3].

3. A generalization of Theorem I may be given as follows:

THEOREM 2. If $G$ is a nilpotent group and $H^{i}\left(G, O_{F}\right)$ is trivial for some integer $i$, then $H^{r}\left(V, O_{F}\right)$ is trivial for all integral values of $r$ and for all subgroups $V$ of $G$.

Proof. It has recently been proved [4] that a finite group $G$ is nilpotent if and only if for every finite $G$-module $M$, any relation $H^{i}(G, M)=0$ implies all relations $H^{r}(G, M)=0(r=0, \pm 1, \pm 2, \cdots)$.

Let $w_{1}, \cdots, w_{n}$ be a normal basis for $F$ over $K$ chosen such that $w_{i} \in O_{F}, i=1, \cdots, m$. Let $\mathfrak{M}=O_{K} w_{1}+\cdots+O_{K} w_{n} . H^{r}(G, \mathfrak{M})=0$ for all $r$ and it follows from this and the exactness of the sequence 


$$
0 \rightarrow \mathfrak{M} \rightarrow O_{F} \rightarrow O_{F / 2 R} \rightarrow 0
$$

that $H^{r}\left(G, O_{F}\right)=H^{r}\left(G, O_{F / \mathfrak{M}}\right)$ for all $r$. Thus if $H^{i}\left(G, O_{F}\right)=0$ for some $i, H^{i}\left(G, O_{F / \mathfrak{M}}\right)=0$. But $\mathfrak{M}$ is of finite index in $O_{F}$, hence $O_{F / \mathfrak{M}}$ is a finite $G$-module. Since $G$ is nilpotent, applying the above stated theorem we have $H^{r}\left(G, O_{R}\right)=0$ for all $r$. Applying Theorem I we obtain Theorem II.

4. Using an argument similar to that in 3 , we see that our counterexample also provides a further example to establish the necessity that $G$ be nilpotent in order for $H^{i}(G, M)=0$ to imply $H^{r}(G, M)=0$ for all $r$ and all finite $G$-modules $M$.

\section{BIBLIOGRAPHY}

1. E. Artin, Kohomologie endlicher Gruppen, Lecture notes, Hamburg, 1957.

2. - Theory of algebraic numbers, Lecture notes, Goettingen, 1959.

3. C. Chevalley, Class field theory, Nagoya Univ., 1953-1954.

4. K. Hoecksman et al., $A$ cohomological characterization of finite nilpotent groups, Arch. Math. 19 (1968), 225-244.

5. E. Weiss, Algebraic number theory, McGraw-Hill, New York, 1963.

6. H. Yokoi, On the ring of integers in an algebraic number field as a representation module of Galois group, Nagoya Math. J. 16 (1960), 83-90.

7. - On an isomorphism of Galois cohomology groups $H^{n}\left(G, O_{K}\right)$ of integers in an algebraic number field, Proc. Japan Acad. 38 (1962), 499-501.

8. - On the Galois cohomology group of the ring of integers in an algebraic number field, Acta Arith. 8 (1963), 243-250.

9. - A note on the Galois cohomology group of the ring of integers in an algebraic number field, Proc. Japan Acad. 40 (1964), 245-246.

Ohio State University 